\title{
Pattern formation in nonlinear diffusion-limited aggregation
}

\author{
Takashi Nagatani \\ College of Engineering, Shizuoka University, Hamamatsu 432, Japan \\ Yoshiyuki Usami \\ Department of Applied Physics, Tokyo Institute of Technology, Tokyo 152, Japan
}

(Received 7 June 1988; revised manuscript received 12 October 1988)

\begin{abstract}
The effect of concentration-dependent diffusivity on diffusion-limited aggregation (DLA) is investigated by a computational method. The growth process is governed by a nonlinear diffusion equation with a concentration-dependent diffusivity. The aggregate grows proportionally to the mass flux on the surface of the aggregate. The development of distinct morphologies is found in nonlinear diffusive pattern-forming systems. When the dependence of diffusivity on the concentration increases, the aggregate crosses over from the DLA fractal to dense aggregation. The crossover phenomenon also occurs with an increase of the concentration difference between the aggregate and the outer boundary. A new dimensionless parameter is found to govern the crossover phenomenon. The morphology diagram is described in terms of the coefficient of the nonlinear part of the diffusivity and the concentration difference between the boundaries. Similarity with pattern formation in electrochemical deposition is discussed.
\end{abstract}

\section{INTRODUCTION}

Pattern formation in systems far away from equilibrium is a subject of considerable current interest. ${ }^{1-7}$ There has been increasing interest in the problem of geometrical structure in the kinetic-growth model. ${ }^{8-12}$ A number of models for cluster formation have been proposed, and a variety of computer simulations have been carried out to investigate the relationships between the cluster geometry and growth mechanisms. The structure of the aggregates strongly depends on the dynamics of the growth process. Examples of pattern formation in a wide variety of diffusive systems include crystal growth, ${ }^{13-17}$ electrochemical deposition, ${ }^{18-20}$ viscous fingering, ${ }^{21-23}$ and dielectric breakdown. ${ }^{24}$ Witten and Sander ${ }^{25}$ proposed the diffusion-limited aggregation (DLA) model to treat the effects of the nonlocal diffusion field. The structure of the aggregate has been analyzed by computational, ${ }^{26,27}$ experimental, ${ }^{13,18,21,24}$ and analytical methods. It has been found that the structure of the aggregate was a fractal. Several analytical attempts, including mean-field theories $^{28-30}$ and renormalization-group methods ${ }^{31-34}$ have been made to derive the fractal dimension. The multifractal structure of the growth probability distribution on a surface of the aggregate was found and analyzed..$^{35,36}$ Vicsek $^{37}$ and Kadanoff ${ }^{38}$ proposed simple generalizations of the DLA model to take into account the stabilizing force of the surface tension. Meakin ${ }^{39}$ investigated the effect of particle drift on DLA with the use of a biased random walker and found a crossover phenomenon from the DLA fractal to the dense aggregation. Voss ${ }^{40}$ proposed the multiparticle fractal aggregation model and found the similar crossover with increasing particle concentration. Nauenberg et al. ${ }^{41}$ analyzed the crossover by using the mean-field approximation in which a finite density of diffusing particles was taken into account. Sarkar and Jensen ${ }^{42}$ studied the evolution of the interface for a one-sided model of directional solidification on a lattice with biased random walkers. Very recently, Grier et al. ${ }^{18}$ and Sawada et al. ${ }^{19}$ found in experiments of the electrochemical deposition that the system was driven from fractal growth, through denseradial aggregation, to dendritic growth by varying the applied potential and the concentration of the electrolyte. Hibbert and Melrose ${ }^{43}$ introduced an experimental technique to prevent convective motion in the electrodeposition. In the electrodeposits on paper, they found only the crossover to the dense-radial aggregation by increasing the applied voltage. The other crossovers were not found.

In the experiments mentioned above, the crossover from the DLA fractal to the dense aggregation was found with increasing the concentration or the applied field. However, the generalized DLA model was not presented to allow for both the applied field and the concentration.

In this paper, we study pattern formation in nonlinear diffusion field. Effects of the concentration-dependent diffusivity on DLA are investigated by the computational method. We propose a simple generalization of the DLA model to take into account the nonlinearity of the diffusion field. The distinct morphologies develop with the increase of a positive nonlinearity of the diffusivity. The nonlinearity acts as a stabilizing force on the interface. The DLA fractal crosses over to the dense aggregation with the increase of the nonlinear effect. In the dimensionless form of the nonlinear diffusion equation, the nonlinear part of the diffusivity is proportional to both the coefficient of the concentration-dependent part and the concentration difference between the aggregate and the outer boundary. The crossover phenomena also occur with the increase of the concentration difference.

The organization of the paper is as follows. In Sec. II we present the basic equation of the nonlinear diffusion field. Our model is given in the discrete version of the 
nonlinear diffusion equation. In Sec. III we present the computational result. The development of the distinct morphologies is shown in the figures. The morphology diagram is found. In Sec. IV our model is applied to the problem of the electrochemical deposition. The similarity to morphologies found in the electrochemical experiments is discussed. Section V presents the summary.

\section{BASIC EQUATION AND MODEL}

We consider the aggregation process growing on a surface in two dimensions. We consider the diffusion field of a component outward from the growing aggregate without surface tension. The diffusivity is dependent on the concentration. The concentration $C$ of the diffusing component satisfies the nonlinear diffusion equation under the quasistationary approximation

$$
\frac{\partial}{\partial x_{i}}\left(D(C) \frac{\partial C}{\partial x_{i}}\right)=0
$$

with

$$
D(C)=D_{0}+\gamma C^{b},
$$

where $D(C)$ indicates the concentration-dependent diffusivity, $D_{0}$ is the concentration-independent part of the diffusivity, $\gamma$ the coefficient of the concentrationdependent part, and $b(>0)$ the order of concentration dependence. We consider the diffusion field within the square with $L \times L$. The aggregate grows from the lower boundary toward the upper boundary. The boundary conditions in the horizontal direction are periodic in order to simulate an infinite half-plane. In the vertical direction the boundary conditions are given by the constant concentrations $C_{u}$ and $C_{s}$, where $C_{u}$ and $C_{s}$ indicate the concentrations on the upper and lower boundaries. We shall consider the case in which the concentration $C_{s}$ on the surface is very small $\left(C_{s}<<1\right)$. To cast the diffusion equation in dimensionless form we use the following units: the concentration difference $\Delta C_{t}\left(=C_{u}-C_{s}\right)$ between the upper and lower boundaries for the concentration, $L$ for distance, and $D_{0}$ for the diffusivity. Then Eq. (1) becomes

$$
\frac{\partial}{\partial \hat{x}_{i}}\left(\hat{D}(\hat{C}) \frac{\partial \hat{C}}{\partial \hat{x}_{i}}\right)=0
$$

with

$$
\widehat{D}(\widehat{C})=1+\left(\gamma / D_{0}\right)\left(\Delta C_{t}\right)^{b} \widehat{C}^{b} .
$$

The boundary conditions are given by $\hat{C}=0$ on the aggregate and $\hat{C}=1$ on the upper boundary, where $\widehat{C}=C / \Delta C_{t}$.

Careted variables are all dimensionless. We find the important dimensionless parameter $\chi=\left(\gamma / D_{0}\right)\left(\Delta C_{t}\right)^{b}$ representing the strength of the nonlinearity of the diffusion field. The nonlinearlity becomes important not only with the large $\gamma$ but also with large $\Delta C_{t}$.

We consider Eq. (2) in the discrete version. The difference equation is given by

$$
\sum_{j} \hat{D}\left(\hat{C}_{j}\right)\left(\hat{C}_{j}-\hat{C}_{i}\right)=0
$$

with

$$
\widehat{D}\left(\widehat{C}_{j}\right)=1+\chi \hat{C}_{j}^{b},
$$

where $j$ represents the nearest-neighbor site of the site $i$, and the summation ranges over all the nearest-neighbor sites. The concentration at the site $i$ is given by

$$
\hat{C}_{i}=\left(\sum_{j} \hat{D}\left(\widehat{C}_{j}\right) \hat{C}_{j}\right) /\left(\sum_{j} \hat{D}_{j}\right) .
$$

Now we consider the simulation of the aggregation process following Eq. (3). The aggregation starts along the lower boundary (see Fig. 1). The concentration field is step by step calculated by solving Eq. (4) under the boundary conditions of the instantaneous aggregate. The growth probability $p_{i}$, added to the segment $i$ connected to the aggregate, is proportional to the local mass flow $\widehat{D}\left(\widehat{C}_{i}\right) \nabla C_{i}$ on the surface of the aggregate. The growth probability proportional to the mass flow is assigned to the perimeter bonds. The interface proceeds to the top according to the growth probability, where the aggregation process occurs one by one. The algorithm of the simulation process is similar to that of the DLA presented by Pietronero and Wiesmann, ${ }^{44}$ except for the nonlinearity.

\section{COMPUTATIONAL RESULTS}

We have carried out calculations for a range of $\chi\left(=\gamma / D_{0}\right)$ values on square arrays of size $200 \times 200$. We restrict ourselves to the case in which the diffusivity increases linearly with the concentration. On the interface of the aggregate the concentration $\widehat{C}$ is zero and on the upper boundary $\widehat{C}=1$. Figure 1 shows the geometrical configuration with the boundary conditions. The range of $\chi$ values is limited by the condition that the diffusivity $D$ is positive,

$$
\chi>-1 \text {. }
$$

We examine the patterns formed by the nonlinear DLA in a range of $-1<\chi \leq 100$. The clusters are grown

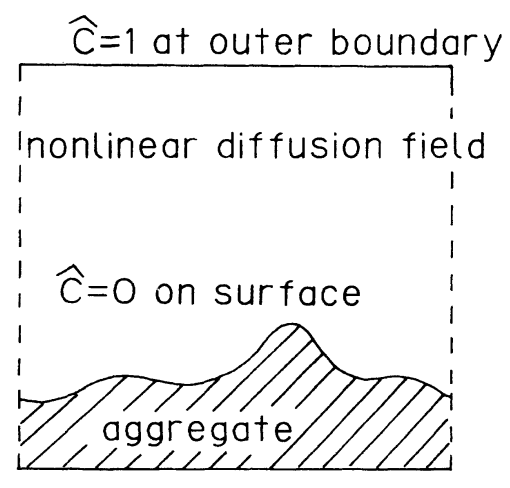

FIG. 1. Schematic representation of the problem used to study the nonlinear effect of the diffusivity on DLA. The aggregate grows to be proportional to the local-mass flux on the surface. The boundary conditions are given by $\hat{C}=0$ on the surface and $\widehat{C}=1$ at the upper boundary. 
(a)

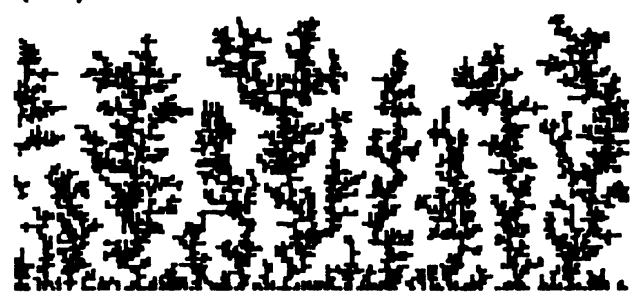

(b)

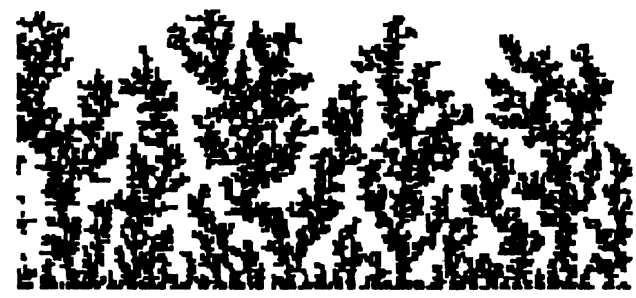

(c)

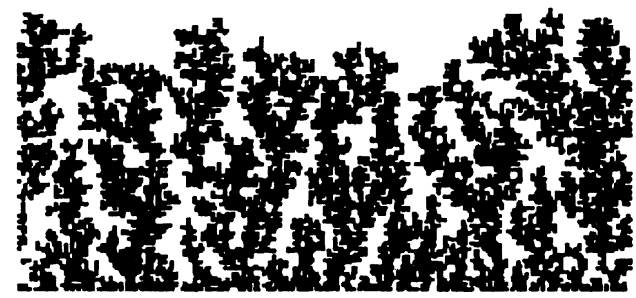

(d)

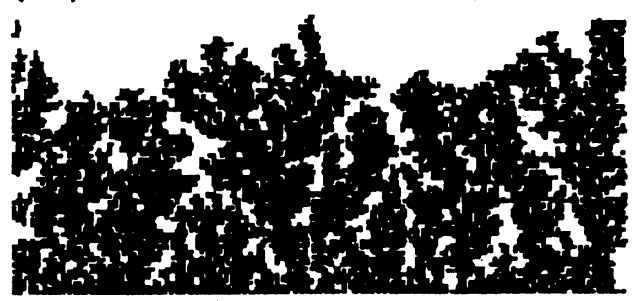

(e)

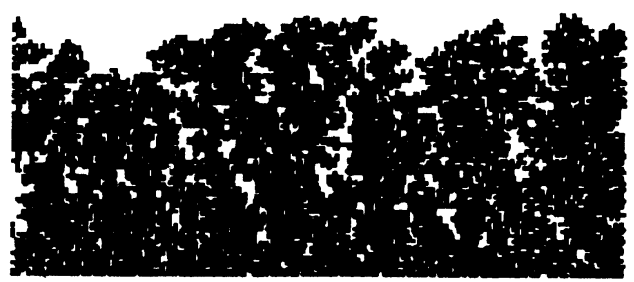

FIG. 2. Typical aggregates grown on a surface. (a) $\chi=0.1$; (b) $\chi=15$; (c) $\chi=25$; (d) $\chi=50$; (e) $\chi=100$. The aggregate crosses over from DLA to dense aggregation with the increase of the strength $\chi$ of the nonlinearity. (a)

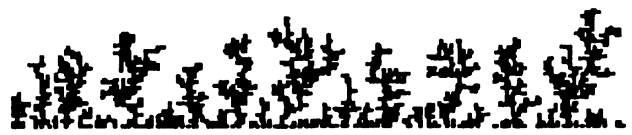

(b)

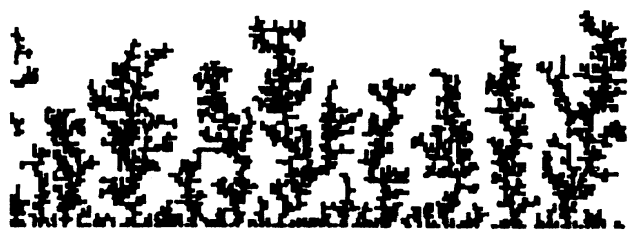

(c)

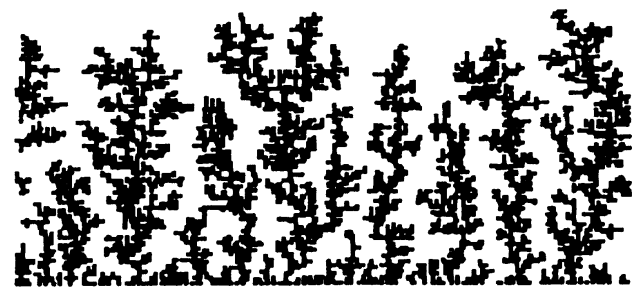

FIG. 3. An evolution process for $\chi=0.1$. (a) 2000 particles; (b) 4000 particles; (c) 5800 particles.

(a)

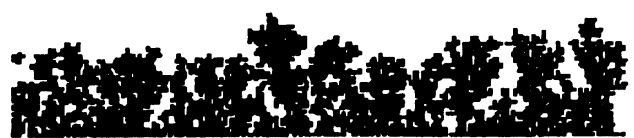

(b)

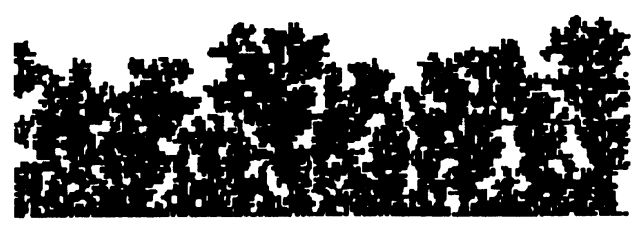

(c)

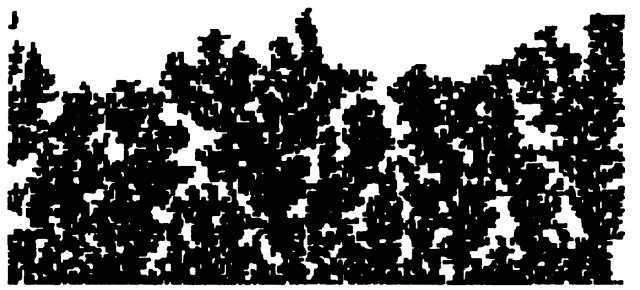

FIG. 4. An evolution process for $\chi=50$. (a) 3500 particles; (b) 7000 particles; (c) 10700 particles. 


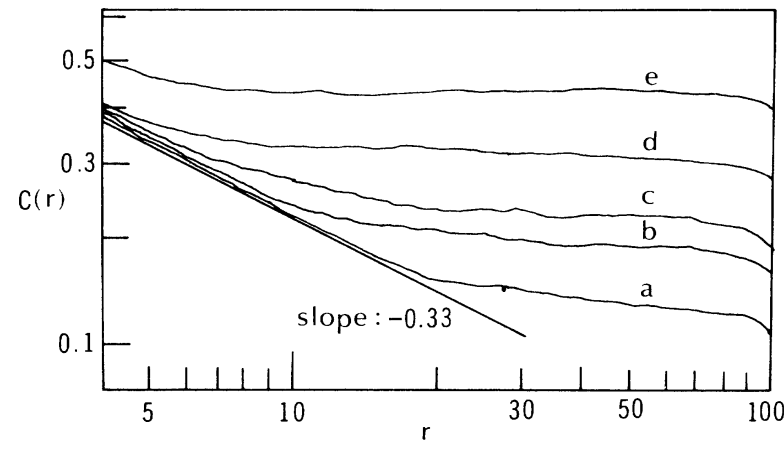

FIG. 5. Density-density correlation functions shown in the form of $\log$-log plots for five clusters grown with the use of different parameters $(\chi)$. (a) $\chi=0.1$, (b) $\chi=15$, (c) $\chi=25$, (d) $\chi=50$, (e) $\chi=100$. The straight line with the slope -0.33 shows the density-density correlation functions for the DLA cluster.

until the height reaches 100 units or the mass of the cluster reaches 13000 particles. The patterns formed in the nonlinear-diffusive system are shown in Fig. 2. The pattern of the cluster shown in Fig. 2(a) was obtained for $\chi=0.1$. Overall the cluster looks like the DLA fractal. Figures 2(b)-2(e) show, respectively, the aggregates for $\chi=15,25,50$, and 100 . The crossover from the DLA fractal to the dense aggregation is found with increasing parameter $\chi$. On the other hand, in the range of $-1<\chi<0$, the pattern remains like a DLA fractal. Figures 3 and 4 show the evolution processes for $\chi=0.1$ and 50. In Figs. 3(a) -3 (c) the aggregates consist, respectively, of 2000, 4000, and 5800 particles. In Fig. 4(a)-4(c) the aggregates consist, respectively, of 3500, 7000, and 10700 particles. The density-density correlation functions are shown in Fig. 5 in the form of log-log plots for five clusters grown with the use of $\chi$ values of $0.1,15,25$, 50 , and 100 . For comparison, the density-density correlation function for DLA is also shown by the straight line with the slope -0.33 . The crossover in the fractal dimensionality of the cluster is found from $D_{f} \sim 1.66$ on short-length scales to $D_{f} \sim 2$ on long-length scales. We define the $\chi$ value separating DLA from the dense aggregation as that at which the dense structure occupies more than $90 \%$ of the aggregate. The $\chi$ value separating the DLA from the dense aggregation is approximately given by

$$
\chi_{c} \simeq 15,
$$

where $\chi=\left(\gamma / D_{0}\right)\left(\Delta C_{t}\right)$. In the limit of $C_{s}=0$, the crossover phenomenon is governed by the two parameters: the coefficient $\gamma / D_{0}$ of the concentration-dependent part of the diffusivity and the concentration difference $\Delta C_{t}$. The crossover line separated between the DLA and the dense aggregation is given by

$$
\left(\gamma / D_{0}\right) \Delta C_{t} \simeq 15 \text {. }
$$

Figure 6 shows the crossover line separated between the distinct morphologies. When the dependence of the diffusivity on the concentration increases, the aggregate crosses over from a DLA fractal to the dense aggrega-

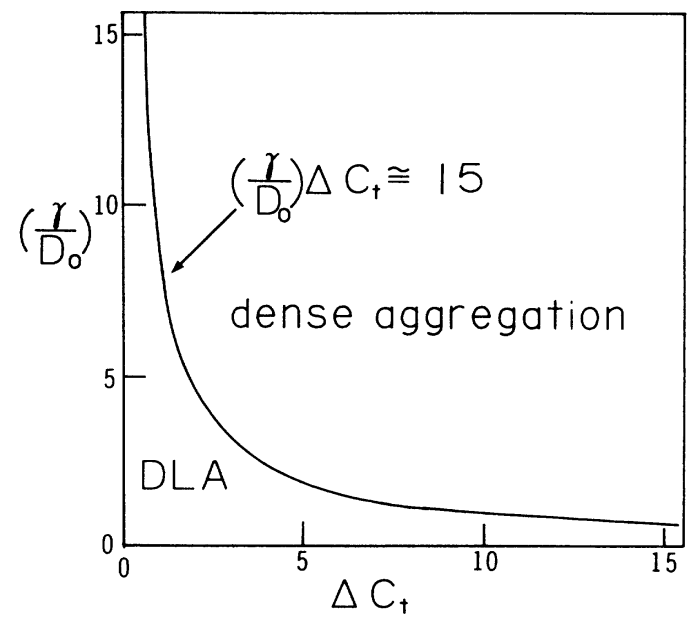

FIG. 6. The morphology diagram. The crossover line separating DLA from the dense aggregation is indicated by the solid line. The DLA fractal crosses over to the dense aggregation with the increase of the coefficient $\gamma$ of the concentrationdependent part of the diffusivity or the concentration difference $\Delta C_{t}$.

tion. The crossover phenomenon also occurs with the increase of the concentration difference between the aggregate and the outer boundary.

\section{APPLICATION TO THE ELECTRODEPOSITION}

We shall apply our result to the development of the distinct morphologies observed in the experiment of the electrochemical deposition. ${ }^{18,19,43}$ In the electrodeposition system the growth rate is controlled by the electric current flowing between the aggregate and the outer boundary. In the quasistationary approximation, the process is determined by the conservation law of current

$$
\frac{\partial}{\partial x_{i}}\left(\sigma(\Phi) \frac{\partial \Phi}{\partial x_{i}}\right)=0,
$$

where $\Phi$ is the electrostatic potential and $\sigma(\Phi)$ indicates the current-dependent conductivity. In the electrodeposition the current increases nonlinearly with the applied voltage. The conductivity increases with the electrolytic concentration of the solution. The current-dependent conductivity will be assumed to be given by

$$
\sigma(\Phi)=\sigma_{0}(C)+\gamma(C) \Phi^{b}
$$

with $\gamma(C) \sim C^{a}$, where $\sigma_{0}(C)$ is the current-independent part of the conductivity, $\gamma(C)$ the coefficient of the current-dependent part, and $\Phi$ the electrostatic potential with the zero potential on the aggregate. $\sigma_{0}(C)$ and $\gamma(C)$ are also dependent upon the electrolytic concentration of the solution. Especially, $\sigma_{0}(C)$ depends strongly on the concentration.

The boundary conditions are given by $\Phi=0$ on the aggregate and $\Phi=\Phi_{u}\left(\Phi_{u}\right.$ a positive constant) at the outer boundary. Our result (7) is applied to the electrodeposition. The crossover line from the DLA fractal to the dense aggregation is given by 


$$
\left[\gamma(C) / \sigma_{0}(C)\right]\left(\Delta \Phi_{t}\right)^{b}=\text { const },
$$

where $\Delta \Phi_{t}$ is the applied voltage between the anode and the cathode. Unfortunately, we cannot account for the data on the conductivity because the dependency of the conductivity on the concentration and the applied voltage was not measured in the electrodeposition experiments. However it is familiar that the conductivity depends strongly upon the concentration and applied field. $\gamma(C) / \sigma_{0}(C)$ will be assumed to be proportional to $\sqrt{C}$ for fitting the experiment. The dependence of the conductivity on the applied voltage is set as $b=1$ for simplicity. If the constant in Eq. (10) is chosen to coincide with the experimental data in the dilute limit, we obtain

$$
C^{0.5} \Delta \Phi_{t} \simeq 1.8
$$

Figure 7 shows the crossover line (11) in comparison with the experimental data by Grier et al. ${ }^{18}$ We find that the nonlinearity of the current-dependent conductivity gives a possible explanation for the crossover from DLA fractal to the dense aggregation. The crossover is also explained by the other mechanism: the finite-diffusion length? In the experiment by Sander et al. ${ }^{45}$ the crossover line agrees with the finite-diffusion length at a relatively dense concentration, but does not in the dilute region. In the experiment by Hibbert and Melrose, ${ }^{43}$ convective motion is not introduced into the diffusion field. However, the crossover occurs. The nonlinear effect may become more important with increasing applied voltage even in a relatively small concentration. Unfortunately, we cannot give the experimental electrochemical evidence. The electrochemical experiments will be expected to measure simultaneously the dependence of the conductivity on both concentration and applied field.

\section{SUMMARY}

We study the effect of the concentration-dependent diffusivity on the diffusion-limited aggregation. We calculate the nonlinear-concentration field by using the relaxation method. We find the crossover phenomenon

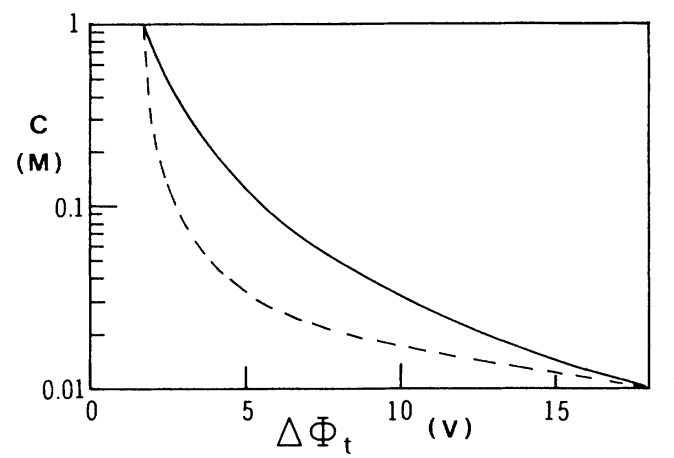

FIG. 7. The morphology diagram for the electrondeposition. the solid and dashed lines indicate the crossover lines obtained by Eq. (11) and the experiment.

from the DLA fractal to the dense aggregation with increase of the nonlinearity of the diffusion field. A new dimensionless parameter $\chi=\left(\gamma / D_{0}\right) \Delta C_{t}$ is found to govern the crossover. The crossover occurs with increasing the nonlinear part of the diffusivity. Also the aggregate crosses over to the dense aggregation with the increase of the concentration difference between the boundaries. The morphology diagram is described in terms of the coefficient of the nonlinear part of the diffusivity and the concentration difference between the boundaries. Application to the electrodeposition is presented. Comparison with the electrochemical deposition experiment is shown. We find that the nonlinearity of the diffusion field becomes important with increasing applied voltage. The nonlinear DLA model presents the generalized DLA model to allow for both applied field and concentration.

\section{ACKNOWLEDGMENTS}

Numerical calculations were performed on the HITAC-S820 computer at the Computer Center of the Institute for Molecular Science (IMS). The authors are grateful for the support provided by the Computer Center of IMS.
${ }^{1}$ J. S. Langer, Rev. Mod. Phys. 52, 1 (1980).

${ }^{2}$ Physica D 12, special issue (1984).

${ }^{3}$ Kinetics of Aggregation and Gelation, edited by F. Family and D. P. Landau (North-Holland, Amsterdam, 1984).

${ }^{4}$ On Growth and Form, edited by H. E. Stanley and N. Ostrowsky (Nijhoff, The Hague, 1985).

${ }^{5}$ Fractals in Physics, edited by L. Pietronero and E. Tosatti (North-Holland, Amsterdam, 1986).

${ }^{6}$ Statistical Physics, edited by H. E. Stanley (North-Holland, Amsterdam, 1986); Physica A 140, special issue (1986).

${ }^{7}$ The Physics of Structure Formation, edited by W. Guttinger and G. Dangelmayr (Springer, Berlin, 1987).

${ }^{8}$ E. Ben-Jacob, N. Goldenfield, J. S. Langer, and G. Schon, Phys. Rev. Lett. 51, 1930 (1983); Phys. Rev. A 29, 330 (1984).

${ }^{9}$ R. C. Brower, D. A. Kessler, J. Koplik, and H. Levine, Phys. Rev. Lett. 51, 1111 (1983); Phys. Rev. A 29, 1335 (1984).

${ }^{10}$ R. Kapral, S. G. Whittington, and R. C. Desai, J. Phys. A 19,
1727 (1986).

${ }^{11}$ F. Family, D. E. Platt, and T. Vicsek, J. Phys. A 20, L1177 (1987).

${ }^{12}$ J. Nittmann and H. E. Stanley, J. Phys. A 20, L1185 (1987).

${ }^{13}$ H. Honjo, S. Ohta, and Y. Sawada, Phys. Rev. Lett. 55, 841 (1985).

${ }^{14}$ H. Honjo, S. Ohta, and M. Matsushita, Phys. Rev. A 36, 4555 (1987).

${ }^{15}$ M. N. Barber, A. Barbieri, and J. S. Langer, Phys. Rev. A 36, 3340 (1987).

${ }^{16}$ Y. Saito, G. Goldbeck-Wood, and H. Muller-Krumbhaar, Phys. Rev. Lett. 58, 1541 (1987).

${ }^{17}$ M. Matsushita, M. Sano, Y. Hayakawa, H. Honjo, and Y. Sawada, Phys. Rev. Lett. 53, 286 (1984).

${ }^{18}$ D. Grier, E. Ben-Jacob, Roy Clarke, and L. M. Sander, Phys. Rev. Lett. 56, 1264 (1986).

${ }^{19}$ Y. Sawada, A. Dougherty, and J. P. Gollub, Phys. Rev. Lett. 
56, 1260 (1986).

${ }^{20}$ D. G. Grier, D. A. Kessler, and L. M. Sander, Phys. Rev. Lett. 59, 2315 (1987).

${ }^{21}$ J. Nittmann, G. Daccord, and H. E. Stanley, Nature (London) 314, 141 (1985).

${ }^{22}$ G. Daccord, J. Nittmann, and H. E. Stanley, Phys. Rev. Lett. 56, 336 (1986).

${ }^{23}$ E. Ben-Jacob, R. Godbey, N. D. Goldenfeld, J. Koplik, H. Levine, T. Mueller, and L. M. Sander, Phys. Rev. Lett. 55, 1315 (1985).

${ }^{24}$ L. Niemeyer, L. Pietronero, and H. J. Wiesmann, Phys. Rev. Lett. 52, 1033 (1984); 57, 650 (1986).

${ }^{25}$ T. A. Witten and L. M. Sander, Phys. Rev. Lett. 47, 1400 (1981); Phys. Rev. B 27, 5686 (1983).

26P. Meakin, Phys. Rev. A 27, 1495 (1983); 27, 2616 (1983).

${ }^{27}$ P. Meakin, H. E. Stanley, A. Coniglio, and T. A. Witten, Phys. Rev. A 32, 2364 (1985); 34, 3325 (1986).

${ }^{28}$ M. Muthukumar, Phys. Rev. Lett. 50, 839 (1983).

${ }^{29}$ M. Tokuyama and K. Kawasaki, Phys. Lett. 100A, 337 (1984).

${ }^{30}$ M. Matsushita, K. Honda, H. Toyoki, Y. Hayakawa, and H. Kondoh, J. Phys. Soc. Jpn. 55, 2618 (1986).

${ }^{31}$ H. Gould, F. Family, and H. E. Stanley, Phys. Rev. Lett. 50,
696 (1983).

${ }^{32}$ M. Kolb, J. Phys. A 20, L285 (1987).

${ }^{33}$ T. Nagatani, J. Phys. A 20, L381 (1987); Phys. Rev. A 36, 5812 (1987).

${ }^{34}$ T. Nagatani, Phys. Rev. A 37, 3514 (1988); 37, 4461 (1988).

${ }^{35}$ T. C. Halsey, P. Meakin, and I. Procaccia, Phys. Rev. Lett. 56, 854 (1986).

${ }^{36}$ C. Amitrano, A. Coniglio, and F. di Liberto, Phys. Rev. Lett. 57, 1016 (1986).

${ }^{37}$ T. Vicsek, Phys. Rev. A 32, 3084 (1985).

${ }^{38}$ L. P. Kadanoff, J. Stat. Phys. 39, 267 (1985).

${ }^{39}$ P. Meakin, Phys. Rev. B 28, 5221 (1983).

${ }^{40}$ R. F. Voss, J. Stat. Phys. 36, 861 (1984).

${ }^{41}$ M.Nauenberg, R. Richter, and L. M. Sander, Phys. Rev. B 28, 1649 (1983).

${ }^{42}$ S. K. Sarkar and M. H. Jensen, Phys. Rev. A 35, 1877 (1987).

${ }^{43}$ D. B. Hibbert and J. R. Melrose, Phys. Rev. A 38, 1036 (1988).

${ }^{44}$ L. Pietronero and H. J. Wiesmann, J. Stat. Phys. 36, 909 (1984).

${ }^{45}$ L. M. Sander, in Ref. 7, p. 257. 